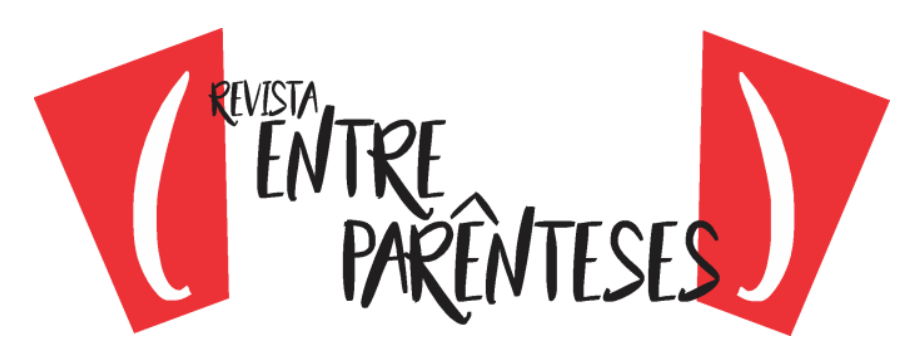

\title{
DESENVOLVIMIENTO PRAGMÁTICO DE BRASILEROS APRENDIENTES DE ESPAÑOL COMO LENGUA EXTRANJERA. ESTRATEGIAS PRAGMALINGÜÍSTICAS DEL ACTO DE PETICIÓN
}

\author{
https://doi.org/10.32988/rep.v1n9.1259 \\ Sonia Toledo Azócar ${ }^{1}$ \\ Pontificia Universidad Católica de Valparaíso, Chile \\ sonia.toledo@pucv.cl
}

\begin{abstract}
Resumen: Este artículo presenta un estudio exploratorio sobre el desenvolvimiento pragmático de brasileros aprendientes de español como lengua extranjera al realizar el acto de petición. Para estos efectos, nos propusimos identificar y analizar las estrategias pragmáticas que seleccionan universitarios brasileros al emitir la acción de pedir en una situación comunicativa académica y en el contexto de una relación interpersonal asimétrica. El corpus está constituido por 20 producciones las que fueron obtenidas a través del método de completación de discurso. Para la clasificación de las estrategias nos valimos de la categorización propuesta por Blum-Kulka, House y Kasper (1989) para el acto en estudio y analizamos en específico el acto nuclear y los movimientos de apoyo a este, como asimismo, los recursos de atenuación e intensificación presentes en su enunciación. Los datos indican que los hablantes brasileros privilegian las estrategias indirectas convencionalizadas al realizar el acto principal y recurren para ello a fórmulas de atenuación morfológica. Respecto de los movimientos de apoyo, es la justificación a la petición la estrategia privilegiada por los lusohablantes quienes optan para su realización a elementos léxicos intensificadores. Se concluye que los sujetos informantes realizan un movimiento estratégico de equilibrio entre recursos atenuadores y elementos intensificadores. Por un lado, deben proteger la imagen tanto del interlocutor como la propia, y por otro, lograr su objetivo comunicativo.
\end{abstract}

Palabras clave: Universitarios brasileros; Desenvolvimiento pragmático; Acto de petición; Estrategias pragmáticas; Español como lengua extranjera.

\section{DESENVOLVIMENTO PRAGMÁTICO DE BRASILEIROS APRENDIZES DE ESPANHOL COMO LÍNGUA ESTRANGEIRA. ESTRATÉGIAS PRAGMALINGUÍSTICAS DO ATO DE PETIÇÃO.}

Resumo: Este artigo apresenta um estudo exploratório sobre o desenvolvimento pragmático dos brasileiros que aprendem o espanhol como língua estrangeira quando apresentam uma petição. Para tal, procuramos identificar e analisar as estratégias pragmáticas que os estudantes universitários brasileiros selecionam quando emitem a ação de petição numa situação de comunicação acadêmica e no contexto de uma relação

1 Sonia Toledo Azócar es profesora de Castellano y Magíster en Literatura Hispánica de la Pontificia Universidad Católica de Valparaíso. También es Doctora en Filología Hispánica de la Universidad de Valladolid (España). Es profesora e investigadora en lingüística aplicada al español como lengua extranjera. Ha efectuado cursos y talleres para alumnos de universidades norteamericanas, europeas, asiáticas y latinoamericanas, realizando además pasantías académicas en España, Suecia, China y Brasil. Además, ha publicado manuales de español y artículos en el ámbito de la adquisición y didáctica del español como segunda lengua. 


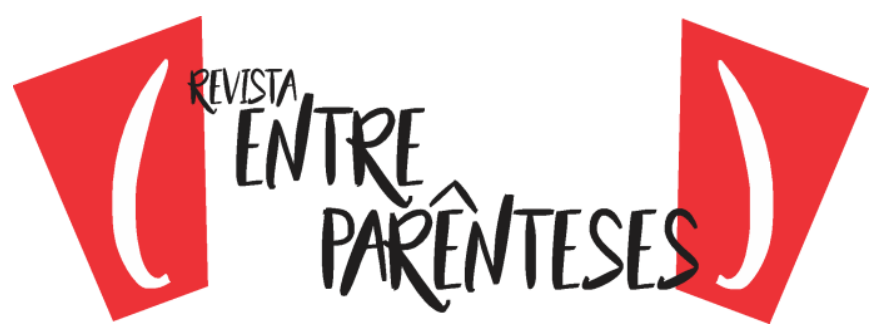

interpessoal assimétrica. O corpus é constituído por 20 produções que foram obtidas através do método de conclusão do discurso. Para a classificação das estratégias utilizamos a categorização proposta por Blum-Kulka, House e Kasper (1989) para o ato em estudo e analisamos especificamente o ato nuclear e os movimentos de apoio ao mesmo, bem como os recursos de atenuação e intensificação presentes na sua enunciação. Os dados indicam que os oradores brasileiros privilegiam estratégias indiretas e convencionalizadas na realização do ato principal e recorrem a fórmulas de atenuação morfológica. No que diz respeito aos movimentos de apoio, a justificação do pedido é a estratégia favorecida pelos lusófonos que optam pela sua implementação para intensificar os elementos léxicos. Conclui-se que os sujeitos informantes realizam um movimento estratégico de equilíbrio entre os recursos atenuantes e os elementos intensificadores. Por um lado, devem proteger a imagem (face) tanto do interlocutor como de si próprios e, por outro, alcançar o seu objetivo comunicativo.

Palavras-chave: Universitários brasileiros; Desenvolvimento pragmático; Ato de petição; Estratégias pragmáticas; Espanhol como língua estrangeira.

\section{PRAGMATIC DEVELOPMENT OF BRAZILIAN STUDENTS OF SPANISH AS A SECOND LANGUAGE. PRAGMALINGUISTICS STRATEGIES OF THE ACT OF PETITION}

Abstract: This article presents a study about the pragmatic development of the Brazilians that learn Spanish as a second language when they accomplish the act of a petition. For this effect, was proposed the identification and the analysis about the pragmatic strategies that select Brazilians college students to emit the action of petition in an academic communicative situation and in context of an interpersonal asymmetric relation. The corpus is constituted by 20 productions obtained through the method of conclusion of speech. For the strategies classification we used the categorization proposal by Blum-Kulka, House e Kasper (1989) for the study act and we analyzed specifically the nuclear act and the support movements for it, as well as the recourses of mitigation and intensification presents in this enunciation. The data indicate that the Brazilian speakers privilege indirect and conventionalized strategies to accomplish the principal act and recover to morphological mitigation formula. In respect of the support movements, the order justification is the strategy favored by the lusophones that choose for the implementation to intensify the lexicon elements. We can conclude the informing subjects accomplish a strategy balance movement between the mitigating resources and the intensifying elements. By one side, they should protect the interlocutor's face as well as themselves and by the other side, get their communication goals.

Keywords: Brazilian university students; pragmatic development, Act of petition, pragmatic strategies; Spanish as a second language.

\section{1- Introducción}

Las transparencias y contrastes entre el español y el portugués se han abordado con profundidad en áreas relacionadas con la habilidad lingüística de brasileros aprendientes de español como lengua extranjera, sin embargo, respecto de su desenvolvimiento pragmático, el desarrollo de investigaciones ha sido menor. En este estudio, entendemos el concepto de desenvolvimiento pragmático como aquel comportamiento verbal e interaccional que presenta un hablante no nativo al seleccionar 


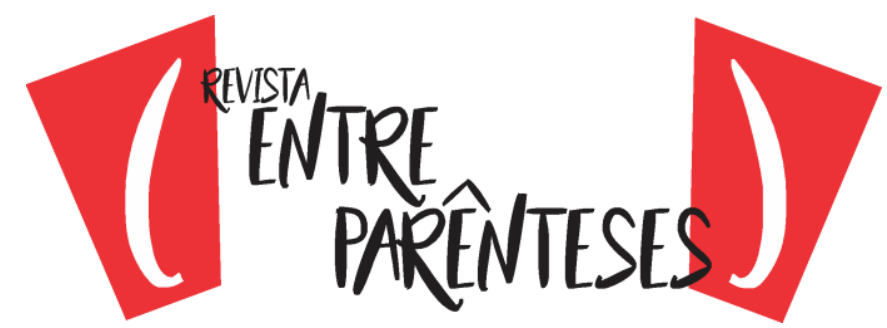

y privilegiar estrategias y recursos pragmalingüísticos para producir un acto de comunicación.

La proximidad tipológica entre el portugués y el español, consideradas dos lenguas "genéticamente emparentadas" (Elizaincin, 1996), ha generado importantes publicaciones con énfasis en el plano léxico. No obstante, como menciona Serrani-Infante (1994) existen críticas dirigidas a investigadores, docentes y creadores de material didáctico por su excesivo interés en formular extensas listas de términos similares y falsos cognados. La autora afirma que en general estos listados aparecen poco contextualizados y adolecen de una justificación sobre su ocurrencia y preferencia por parte de hablantes nativos. Este mismo punto es abordado por Moriondo Kulikowski y Maia González (1999) quienes señalan que en ocasiones estos glosarios son presentados como sinónimos, sin aludir a sus sutilezas semánticas y con una aproximación obsoleta de la lengua como un producto estático "sin historia y sin memoria" (p.13). A los contrastes léxicos (heterosemánticos), se suman las divergencias y similitudes morfológicas, algunos aspectos fónicos (heterofónicos) y en menor grado diferencias en las estructuras sintácticas cuyo acercamiento a través del Análisis Contrastivo ha repercutido en los enfoques metodológicos utilizados históricamente para la enseñanza de español como lengua extranjera o segunda lengua. Tal como constatan Causse y Rodrigues de Almeida (2014), los existentes estudios contrastivos de fenómenos lingüísticos español-portugués en escasas ocasiones han favorecido la descripción de la lengua en uso.

Según Rienda y Nieto (2018) "la semejanza interlingüística consigue crear una situación de partida muy peculiar, una especie de plataforma que sitúa al aprendiz en un nivel bastante más avanzado del que tendría en el caso de lenguas con mayor distancia" (p.112). El aprendiente brasilero de español cuenta entonces con una especie de pasaporte o punto de partida para transferir estructuras de su lengua materna a la lengua meta.

Los estudios coinciden en que la cercanía interlingüística, portugués y español, presenta ventajas para la comunicación puesto que agiliza la comprensión y producción 


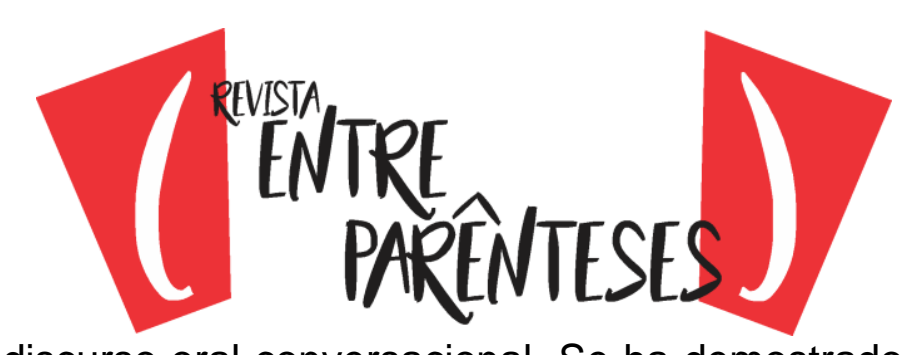

principalmente del discurso oral conversacional. Se ha demostrado que esto puede ser favorable en los primeros estadios de adquisición del español. Sin embargo, una vez que el lusohablante alcanza un nivel más avanzado de dominio del español, o bien, debe realizar actividades escritas de mayor complejidad, por ejemplo, en un contexto académico, esta praxis no es suficiente para adquirir las particularidades de la lengua objeto. Respecto de los aprendientes avanzados de español, Vigón Artos (2005) puntualiza que "la mayoría de los estudiantes lusófonos no consigue avanzar en el proceso de aprendizaje, sino que llega un momento que fosilizan su interlengua, haciendo de ella un sistema gramatical coherente, desarrollado y con reglas propias" (p.661).

Ahora bien, como ya se ha detallado en líneas anteriores, en el ámbito de las competencias sociocultural y pragmática, las transferencias positivas e interferencias españolas y portuguesas no se han investigado con tanta detención aun cuando estas habilidades pueden tener mayor impacto en la comunicación intercultural. Se debe reconocer que esta situación ha cambiado los últimos años y se han desarrollado desde las universidades brasileras interesantes trabajos de cortesía y descortesía verbal, como asimismo, aportadores estudios sobre la realización de algunos actos de comunicación. En efecto, la importancia de la competencia pragmática ha abierto una serie de líneas de investigación que suponen un avance en los estudios de español como lengua extranjera y en sus proyecciones metodológicas. Otero Bravo (2005) en su texto "Competencia pragmática e interferencias culturales en la enseñanza de español para alumnos brasileros", señala que la cercanía hace que sea difícil alcanzar la conciencia de las diferencias pragmáticas que hay entre las dos lenguas (p.15). La proximidad de la cultura brasilera con otras culturas latinoamericanas, puede eclipsar 0 atenuar los contrastes entre ambas lenguas. Es importante, por tanto, detenerse a reflexionar si esta proximidad es solo a nivel de la estructura formal de la lengua, o bien, compromete también a aspectos propios del desenvolvimiento pragmático de los hablantes no nativos de nuestra investigación.

En el presente trabajo, nos preguntamos cuáles son las estrategias pragmáticas que seleccionan los universitarios brasileros aprendientes de español para 


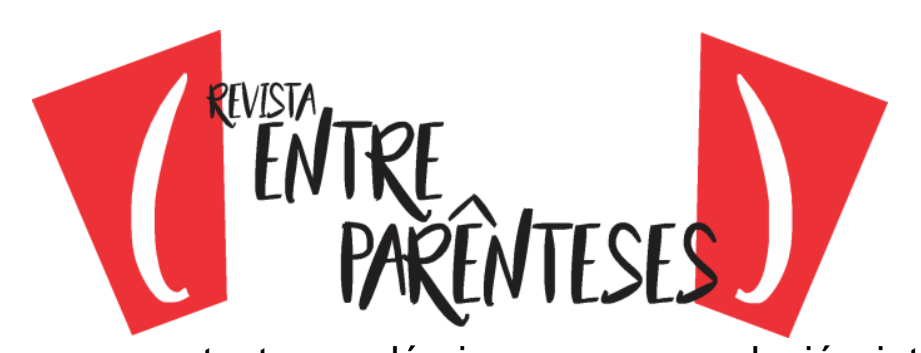

emitir una petición en un contexto académico y en una relación interpersonal vertical, alumno (hablante) - profesor (interlocutor), en la que el interlocutor es quien ostenta el poder. Con la finalidad de responder a esta interrogante realizamos un estudio exploratorio cuyo objetivo central es clasificar y analizar las estrategias pragmáticas y movimientos utilizados por los sujetos informantes de este estudio. Concebimos el concepto de estrategias como aquellas funciones pragmáticas desplegadas por los hablantes para lograr ciertos propósitos comunicativos. Para la clasificación de estas, recurrimos a la taxonomía propuesta por Blum-Kulka, House y Kasper (1989) y analizamos en específico el acto nuclear y los movimientos de apoyo seleccionados por los hablantes para alcanzar su meta comunicativa. Para las autoras, el acto nuclear (head act) es la petición principal que expresa la fuerza ilocutiva del acto y constituye el elemento mínimo para que funcione y se clasifique el acto como tal. Este se puede enunciar como una petición directa o indirecta. El acto nuclear es acompañado por movimientos de apoyo (supportive moves) que son los elementos que aparecen antes o después del acto central y que lo modifican, lo atenúan o lo intensifican.

Con esta investigación se espera en términos generales, aportar a la reflexión sobre el valor del componente pragmático en la comunicación interpersonal e intercultural respecto de la realización de actos de comunicación, y en forma especial, contribuir al conocimiento de la estructura y elementos constitutivos del acto de la petición en español que, si bien ha sido una de las actividades de comunicación más estudiadas en diversas lenguas, con diversos perfiles de hablantes y métodos, en el caso de los lusohablantes de la variante brasilera, aún queda por investigar en diversos contextos situacionales e interpersonales.

\section{2 - Lineamientos teóricos}

\section{1- Habilidad pragmática. El caso de brasileros aprendientes de español.}

La habilidad pragmática constituye uno de los ejes centrales de la visión comunicativa de la lengua (Bachman, 1990; Bachman y Palmer, 1996) y se visualiza 


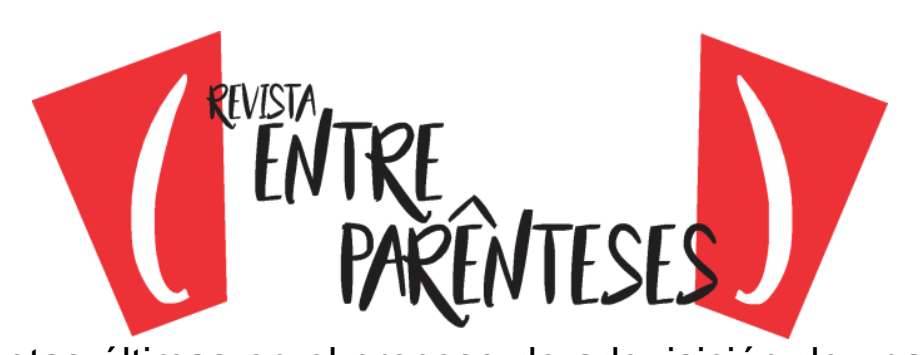

como una de las metas últimas en el proceso de adquisición de una segunda lengua o lengua extranjera. Kasper (1997) la resumen como el conocimiento de una actividad de comunicación y su habilidad de realizarla de acuerdo al contexto. Es más, esta competencia encierra la capacidad de los hablantes de ajustar sus producciones a los efectos que intenta lograr, al contexto, como también a su interlocutor (Toledo, 2018), es decir, a las relaciones interpersonales entre los participantes de una interacción comunicativa, como es la relación de distancia social, poder, cercanía o solidaridad.

Respecto del español y portugués, Moriondo Kulikowski y Maia González (1999) plantean que "las maneras diferentes de organización que no son solo sintácticas, morfológicas o semánticas, nos colocan en lugares diferentes para enunciar y significar y nos llevan a adoptar diferentes estrategias discursivas" (p.5). En efecto, la interpretación o lectura desde la óptica de la cultura brasilera y su lengua genera aciertos, por cierto, no menores, pero también puede resultar en algunas inadecuaciones pragmáticas o fallos pragmáticos, según la conceptualización de Thomas (1983). Según la autora, estos aluden a la incapacidad de entender la intención subyacente de lo enunciado. A nivel de producción dicha incapacidad puede incluir fallos a la norma de la lengua meta no solo en aspectos propiamente lingüísticos sino también en aquellos que afectan la adecuación al contexto, o bien, a los patrones socioculturales que caracterizan la comunidad meta.

Los desajustes pragmáticos pueden tener impacto en la comunicación interpersonal y pueden exacerbar los estereotipos negativos puesto que el interlocutor podría interpretar las producciones pragmalingüísticas del hablante como rudas e incluso descorteses y en el caso de los brasileros, por el contrario, excesivamente corteses. Se debe considerar que la percepción de la adecuación comunicativa tiene algunas diferencias y especificidades de acuerdo a cada sociocultura. Los patrones y guiones de comportamiento pragmático que rigen las interacciones en una comunidad social, generalmente no son iguales o por lo menos, no exactamente iguales. A esto se suma que no siempre las reglas pragmáticas son explícitas o transparentes, razón por la cual 


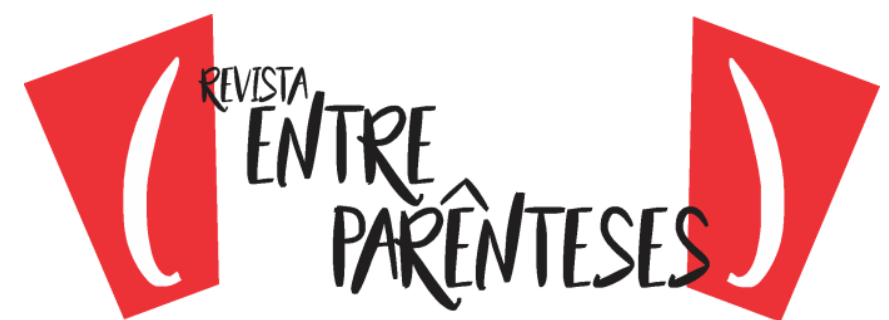

su enseñanza y aplicación en el aula de español como lengua extranjera, debe tener un espacio de relevancia.

En el marco del Análisis Contrastivo (Lado,1957) se planteó inicialmente la teoría de que la proximidad entre lenguas facilita el aprendizaje de una de ellas, pero posteriormente se pudo corroborar con estudios empíricos que en ciertas situaciones comunicativas se producían más interferencias que transferencias positivas. La confianza de los lusófonos, en algunos contextos de comunicación, los lleva a transferir literalmente el comportamiento pragmático de su lengua materna a la lengua meta. En palabras de Otero Bravo (2005) "la cercanía hace que sea difícil alcanzar la conciencia de las diferencias pragmáticas que hay entre las dos lenguas" (p. 15) de ahí que esta transparencia entre ambas lenguas -ilusoria según algunos autores-, es un desafío en la enseñanza y aprendizaje del español como lengua extranjera y que puede presentar complejidades incluso en niveles avanzados de dominio de la lengua meta. Al respecto Moriondo Kulikowski y Maia González (1999) manifiestan que "la [..] cercanía facilita presuposiciones que después no se confirman, pero que son muy resistentes (todos entienden todo), en síntesis, crea o permite una transparencia engañosa que se presta a innumerables equívocos" (p.15) Las inadecuaciones o desajustes pragmáticos son percibidos en ocasiones como falta de cortesía.

\section{2 - Cortesía verbal y actos de comunicación.}

La cortesía se define como "una actividad comunicativa cuya finalidad es quedar bien con el otro" (Bravo, 2005, p. 33). Como plantea Briz (2007) "se visualiza como un fenómeno de acercamiento o aproximación al otro" (p. 2). Se trata de un modo de comportamiento pragmático que permite mantener el orden armonioso en las relaciones interpersonales, evitando lo más posible el conflicto entre los interactuantes y dando cuenta de las expectativas de actuación de cada comunidad de habla.

Dentro de los estudios actuales de cortesía verbal del grupo lusohablantes, sobresalen el de Gallardo Moraes (2013) "As manifestações de cortesia encontradas em 


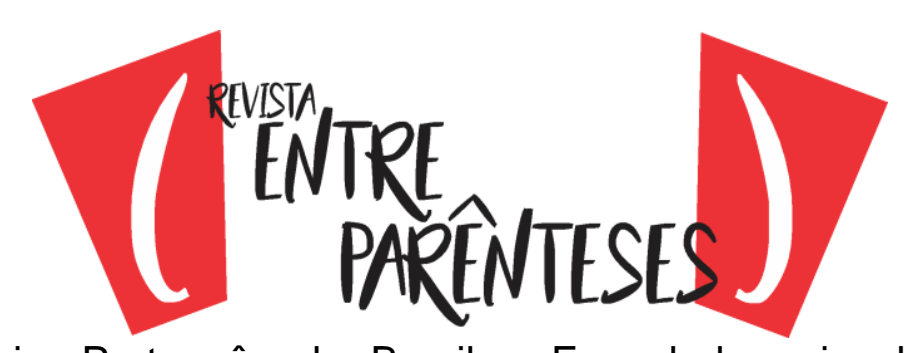

e-mails empresariais. Português do Brasil e Espanhol peninsular: semelhanças e contrastes”; el de Cathcart y Almeida (2014) “¡No! La negación en el español de Cuba y en el portugués brasileño bajo la perspectiva pragmática de la cortesía" y el estudio de Da Silva (2008) "Cortesía y discurso académico" quien trabaja específicamente sobre las relaciones de jerarquía y solidaridad en la relación de profesor-alumno en una clase. También en un contexto académico, Elisa Lopes Rodrigues y Adriana Marcelle de Andrade (2012) se centran en encuentros orales con fines de estudio y realizan un aportador trabajo contrastivo portugués de Brasil y español de Chile.

Ahora bien, la cortesía se produce en un determinado contexto sociocultural, cuyos componentes garantizan la presencia o ausencia de adecuación en los actos de habla. Mientras el acto sea más amenazante a la imagen del interlocutor (Goffman, 1967), el hablante activa un número mayor y más eficaz de estrategias pragmáticas que dan cuenta de su desenvolvimiento pragmático.

Los últimos años se han evidenciado estudios que se han centrado en estudios contrastivos entre lusohablantes e hispanoparlantes respecto de la realización de actos comunicativos. Andrade (2016) examina específicamente los actos asertivos y concluye que los brasileros manifiestan una tendencia a la imagen de cordialidad y cooperación en la construcción de su autoimagen en interacciones académicas grupales. Por su parte, Serrani-Infante (1994) analiza el acto de rechazar un pedido y concluye que los brasileros son menos imperativos que los hispanoparlantes al recurrir a construcciones modalizadores y atenuaciones. A la misma conclusión llega Da Silva (2008) quien plantea que los brasileros son corteses y muy atenuantes y usan muchos rodeos para realizar los actos de rechazar una invitación y pedir un favor.

En relación con la cortesía verbal emergen las categorías pragmáticas de la atenuación y la intensificación. Briz (2005) le asigna a la atenuación la función de "minimizar de forma directa el contenido proposicional de lo que se dice [...] e indirectamente el decir" (p. 81). Briz y Albelda (2013) agregan que la atenuación es un mecanismo estratégico de distanciamiento lingüístico del mensaje y al mismo tiempo de 


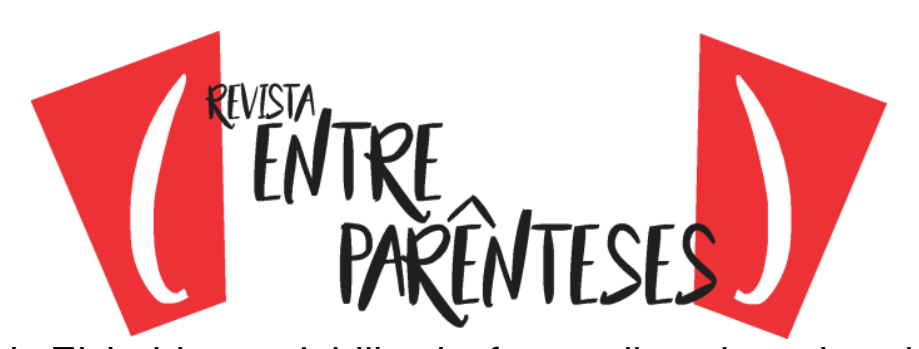

acercamiento social. El hablante debilita la fuerza ilocutiva, de tal manera que logra distanciarse de su mensaje para acercarse social y afectivamente.

Por su parte, la intensificación tiene como papel aumentar la fuerza ilocutiva de un acto de habla. El hablante utiliza elementos intensificadores para reforzar la verdad de lo enunciado (Albelda, 2005, p. 230), "bien modificando superlativamente la cantidad o la cualidad de lo dicho o bien aumentando el grado de fuerza ilocutiva de un acto de habla" (Albelda y Barros, 2018, p. 41). Estas dos categorías pragmáticas serán fundamentales en la configuración de una petición y su grado de dirección.

\subsection{El acto de petición}

Se considera clásicamente como un acto directivo (Searle, 1969) en tanto se trata de una actividad verbal que persigue que el interlocutor haga, o bien, no haga algo que beneficie al hablante. Se clasifica como un acto que amenaza la imagen social (face según el estudio clásico de Goffman, 1967) del interlocutor. El hablante invade su territorio, instando con mayor o menor fuerza o con mayor o menor directividad, un cambio del actuar de su interactuante. Dada la naturaleza impositiva de la acción de pedir es que los hablantes persiguen mitigar el carácter directivo del acto, "suelen recurrir a más estrategias corteses que algunos actos que son puramente asertivos, descriptivos e informativos" (Albelda y Barros, 2018, p.35). El acto de pedir está sujeto en su enunciación a un grado mayor o menor de indirección, es así como una petición se da de manera indirecta cuando la fuerza locutiva del enunciado no coincide con la fuerza ilocutiva de dicho enunciado, en otras palabras, la intención del mismo no se explicita.

Ahora bien, las peticiones han sido ampliamente estudiadas en segundas lenguas y en estudios contrastivos dado que, como las caracterizan Blum- Kulka y Olshtain (1986), son actos "fascinantes" porque dan lugar a una gran variabilidad en las formas y en los modos en los que estas se realizan. En el caso de estudios de lusohablantes, cabe mencionar los trabajos de Gallardo Moraes (2013) quien analiza las manifestaciones de cortesía en peticiones de correos electrónicos en el contexto de una empresa de Brasil y 


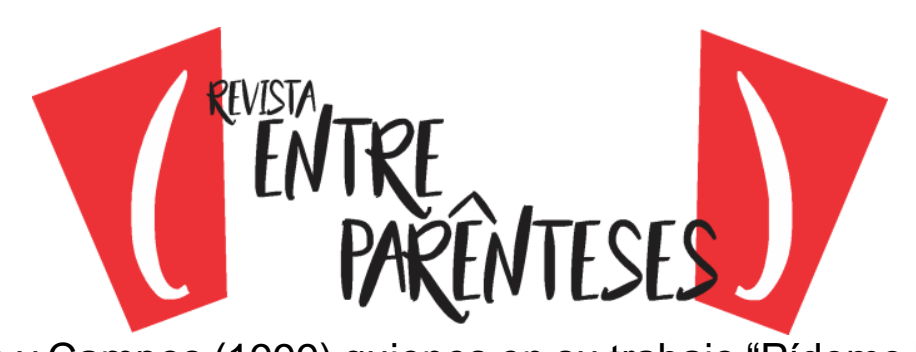

España y de Duarte y Campos (1999) quienes en su trabajo "Pídeme lo que quieras, pero dime por favor! Las dificultades pragmáticas de los aprendientes brasileros al usar el Imperativo en español" examinan las complicaciones de los lusohablantes al utilizar formas del modo imperativo y concluyen que en la cultura brasilera se privilegia sugerir o pedir, en vez de ordenar muy fuertemente. Los hablantes recurren a estrategias atenuadoras como formas modales, o si es imperativo, se suaviza con minimizadores léxicos. Para las autoras, sería poco usual escuchar a un brasilero emitir el siguiente enunciado: Traga-me uma cerveja y optaría por formas atenuadoras del tipo: Podería me trazer uma cervejinha, por favor.

En relación también a la cortesía, Moriondo Kulikowski (2010) en su texto "Los estudios de la cortesía. Subsidios para su ubicación en el aula de E/LE", plantea como hipótesis que el portugués de Brasil recurre a un número mayor de fórmulas atenuadoras que el español peninsular y que la percepción de lo que puede ser una actividad cortés no siempre coinciden en una cultura y otra. Las expectativas y reglas de cortesía consensuadas por una comunidad para realizar actos de comunicación, aparecen latentes pero muchas veces, invisibles. Esto hace que, en el caso de los brasileros, a pesar de estar dentro de un mismo código cultural (lo latinoamericano), la percepción de lo adecuado en las relaciones verticales y de distancia social, pueden presentar algunas diferencias.

\section{3- Metodología}

El corpus está constituido por 20 realizaciones en español de hablantes brasileros. Identificamos y analizamos las estrategias y movimientos pragmáticos que despliegan estos aprendientes de español al emitir una petición. En la tarea de recopilación del corpus, se optó por la completación de discurso (Discourse Completion Test), instrumento privilegiado para el estudio de realización de actos de habla y que tiene un extenso historial en trabajos de Pragmática Intercultural e Interlingüística del acto de petición. Este método de recogida de datos presenta puntos a favor para el presente 


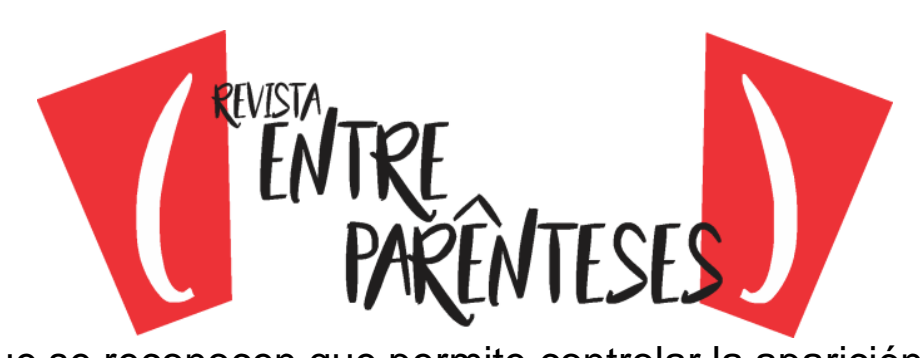

trabajo, entre los que se reconocen que permite controlar la aparición del acto en estudio en un contexto específico y en forma especial que se puede manejar de mejor manera las variables sociales e interpersonales involucradas. Además, las realizaciones obtenidas a través del DCT pueden ayudar como punto de partida para clasificar los tipos de estrategias que se pudiesen dar en las interacciones de habla espontánea.

El corpus fue recolectado el año 2018 en universidades del estado de Paraíba. Se recopiló de forma escrita y presencial por profesores de español en sus respectivas universidades y cursos de español.

Se propuso la siguiente situación comunicativa para realizar el acto de petición: usted es un estudiante universitario, tiene un problema y debe faltar a un examen final. Le pide a su profesor rendir su examen en otra fecha, ¿qué le diría?

Se les pidió a los informantes que respondiesen por escrito y en español tal como lo harían en una situación real. No hubo tiempo límite para realizar esta tarea comunicativa.

Los sujetos informantes son universitarios, hombres y mujeres de entre 19 a 24 años. Un número significativo son estudiantes de la carrera de español. Corresponden a un nivel intermedio avanzado de manejo del español (B2) que tienen a lo menos 4 semestres de instrucción formal universitaria.

Respecto del método de análisis, recurrimos, como se señaló en párrafos anteriores, a la categorización del acto de petición de Blum Kulka, House y Kasper (1989) en su proyecto Cross-Cultural Study of Speech Act Realization Patterns. Las autoras propusieron un repertorio de estrategias de acuerdo al nivel de directividad de la emisión de las peticiones: las directas, a través de las cuales el hablante expresa directamente la petición, mediante formas imperativas, expresiones de obligación, de necesidad o deseo; las estrategias indirectas convencionales que incluyen peticiones que se formulan como sugerencias o preguntas preparatorias; y las indirectas, a modo de alusiones fuertes o débiles. Una estrategia pragmática es más directa que otra si la intención ilocutiva es más aparente, es decir, si el proceso interpretativo para llegar de lo que se dice a lo que se quiere decir es más explícito. El estudio clásico de Blum-Kulka et al. arroja como resultado 


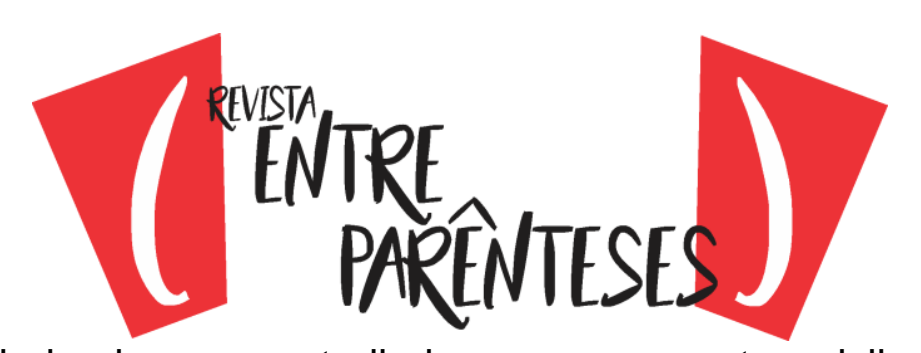

que las mayorías de las lenguas estudiadas en su proyecto, privilegian las estrategias indirectas convencionalizadas, es decir, aquellas formas idiomáticas estandarizadas en actos de habla indirectos, llamadas así puesto que tienen la propiedad de mantener su significado literal y convencional.

La contribución de las autoras es innegable en el ámbito de la cortesía verbal ya que proponen una clasificación del acto de la petición, el que se construye en base a: i) alertadores (alerters), elementos que abren la petición y que funcionan como llamados de atención al interlocutor, generalmente bajo la forma de vocativos $u$ otras formas apelativas; ii) el acto nuclear (head act) que consiste en la petición principal que expresa la fuerza ilocutiva del acto y constituye el elemento mínimo para que funcione y se clasifique el acto como tal. Este se puede clasificar como una petición directa o indirecta; y iii) los movimientos de apoyo (supportive moves) que acompañan el acto nuclear y son los elementos constituyentes que aparecen antes o después del acto central y que lo modifican, lo atenúan o lo intensifican.

\section{4- Resultados y discusión}

En este estudio exploratorio se ha analizado, como punto de partida, el acto nuclear o principal y los movimientos de apoyo que influyen en su articulación.

En primer lugar, presentamos algunos ejemplos de las realizaciones de nuestros informantes con la finalidad de reconocer las estructuras anteriormente referidas.

Tarea comunicativa: usted es un estudiante universitario. Tiene un problema y debe faltar a un examen final. Le pide a su profesor rendir su examen en otra fecha ¿qué le diría?

HnN2: Profesor, tengo un compromiso en familia muy importante y que no puedo perder (movimiento de apoyo) ¿Puedes por favor marcar otra fecha para hacer el examen final o hacer una reposición? (acto nuclear). 


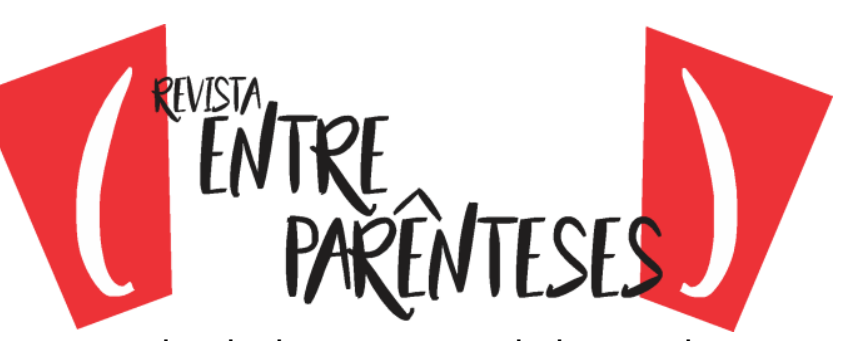

HnN14: Profesor, disculpe, necesito decir que no puedo hacer el examen en la fecha que usted has* elegido (movimiento de apoyo) ¿puedo hacerla en otro momento?" (acto nuclear).

Respecto del acto nuclear, se da una tendencia significativa al uso de estrategias indirectas convencionalizadas, de acuerdo a clasificación de Blum-Kulka et al. (1989). Se observa una presencia muy importante de la petición a través del verbo modal poder, que ejemplificamos en los siguientes enunciados, en especial desde la perspectiva del hablante:

HnN20: Profesor, el próximo día voy a resolver un problema personal ¿puedo hacerlo antes o después de ese día? (acto nuclear).

HnN14: Profesor, disculpe, necesito decir que no puedo hacer el examen en la fecha que usted has* elegido ¿puedo hacerla en otro momento? (acto nuclear).

Cabe mencionar que en muchas de las realizaciones, el verbo poder se estructura en tiempo presente y no en la forma condicional (podría) que marcaría una mayor indirección de la petición.

En el siguiente ejemplo: "Profesor, quería pedirle disculpas porque no voy a poder estar aquí el día del examen final, puedo hacerlo antes o después, ¿qué será mejor para usted?", se puede observar que el hablante recurre a un reforzamiento de su petición con otra pregunta la que atenúa el acto y protege la imagen del interlocutor, evitando ser impositivo con su petición y reforzando que la decisión, en definitiva, está en manos del profesor.

A la estrategia pragmática estructurada con el verbo poder la sigue en frecuencia, la presencia de realizaciones de la petición con el verbo volitivo querer, atenuando así la forma verbal que lo continúa, como se puede observar en los siguientes casos:

HnN 12: Hola profesora, yo quería pedir para* rendir el examen más tarde. Yo tengo un problema muy personal que debo resolver. Puedo rendir el examen la próxima semana por favor?. 


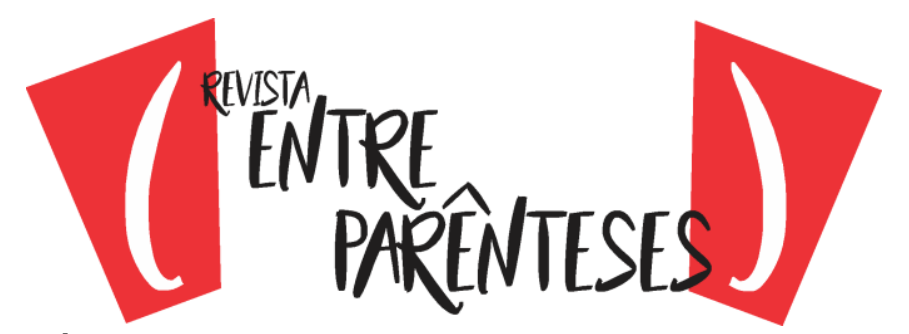

La atenuación de lo enunciado es mayor en la siguiente muestra dado que el verbo saber es modificado por el verbo querer y por la realización minimizada a través de la pregunta condicionada por el si-condicional- y la presencia del elemento atenuador por favor en la posición estratégica de cierre del enunciado:

HnN1: Disculpa* señor, pero tengo un problema personal muy grande y quiero saber si es posible darme otro trabajo, tal vez más difícil para hacer a casa, o una fecha para rendir el examen por favor?

En la siguiente muestra el hablante recurre a la emisión de dos peticiones:

HnN12: Hola profesor, yo quería pedir para* rendir el examen más tarde. Yo tengo un problema muy personal que debo resolver. ¿Puedo rendir el examen la próxima semana por favor?.

A nuestro juicio esto responder a que el hablante puede haber percibido que la primera emisión del acto no fue demasiado explícita o directa "Yo quería pedir para rendir el examen más tarde", al cierre de su intervención entonces reanuda un movimiento de petición: "Puedo rendir el examen la próxima semana por favor?". A nuestro juicio constituye el acto nuclear propiamente tal, intensificando su fuerza con la primera petición.

Ahora bien, en lo que se refiere a los movimientos de apoyo, se puede observar en gran parte de las realizaciones de los informantes una presencia frecuente de dos tipos de modificadores del acto nuclear: 1) la justificación de la petición y 2) la explicitación o explicación de su problema y como se ejemplifica en los siguientes enunciados:

HnN3: Profesor, estoy con problemas con mi familia, que está muy complicada (1) y unfortunadamente ${ }^{\star}$ no puedo hacer el examen final (2) ¿Puedo hacer en otro día?. 


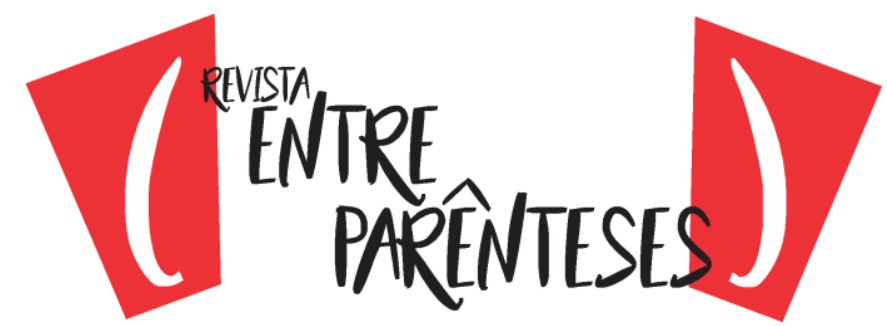

Consideramos que ambas estrategias emergen a modo de una ecuación de movimientos entrelazados: justificación + explicación. "Han pasado muchas cosas personales conmigo así que no puedo hacer la prueba".

Los movimientos que preceden al acto nuclear funcionan como una ecuación argumentativa para preparar al interlocutor a la petición, de ahí que se construyan algunas de ellas con fórmulas intensificadoras: "Yo tengo un problema muy personal", "Tengo un compromiso urgente" y "Tengo un problema personal muy grande".

Otro movimiento de apoyo que aparece en el corpus, con menor frecuencia si se compara con los modificadores anteriores, es el conocimiento de lo instituido (uso del verbo saber) y que funciona a nuestro entender con perseguir empatía con el interlocutor:

HnN7: Profesor, sé que la prueba está fijada hace muchos días, pero estoy con un problema personal) [...]

HnN6: Profesor, estoy con un problema personal y no puedo hacer la prueba. Sé que has ${ }^{*}$ fijado el examen hace bastante tiempo [..]

HnN15: [...] Sabiendo que el señor ha marcado hace bastante tiempo. te pido otra oportunidad para realizar el examen, por favor.

En la mayoría de los casos analizados, se puede apreciar que los movimientos de apoyo preceden al acto nuclear. La elección del hablante de antecederlo responde a una estrategia de preparar al interlocutor para la petición venidera. Incluso, la organización a modo de una seguidilla de movimientos de apoyo, funciona como un intensificador de esta argumentación cuya finalidad es lograr su meta comunicativa, que no es otra que el profesor acceda la petición del alumno.

HnN15: Profesor, tengo un compromiso urgente (modificador 1) $\mathrm{e}^{*}$ no voy poder realizar el examen final en esta fecha (modificador 2). Sabiendo que el señor ha marcado hace bastante tiempo (modificador 3 ), te ${ }^{\star}$ pido otra oportunidad para realizar el examen, por favor (acto nuclear). 


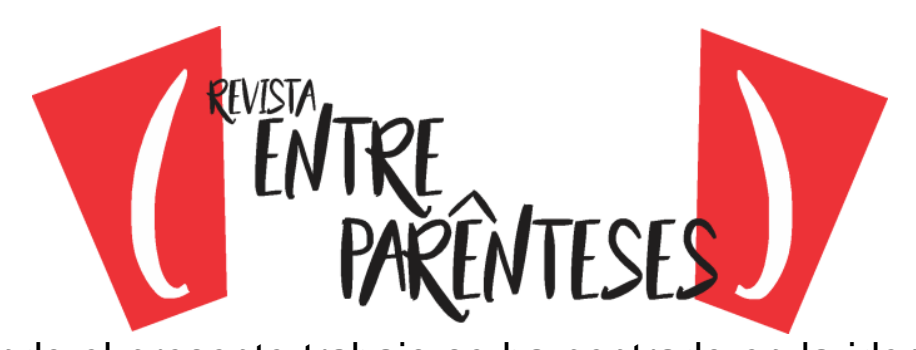

Aun cuando el presente trabajo se ha centrado en la identificación y análisis preliminar del acto principal y los modificadores a este, revisamos también los alertadores que abren la interacción. La mayoría de los informantes recurren al vocativo que da cuenta del estatus o rol social y académico del interlocutor, y sitúa al hablante y oyente en la relación de verticalidad que los caracteriza, tal como se puede apreciar en los siguientes ejemplos: "Profesor, infelizmente no poderé* estar acá durante el examen final", "Perdón profesor, siento informar que non* podré hacer el examen final en esta fecha".

Algunos informantes complementan este nominativo con llamados de atención: "Disculpe profesor, pero tengo un problema muy grande [..]"; "Perdón profesor, lo siento informar que non* podré hacer el examen final en esta fecha”. A nuestro juicio, no corresponde a un acto de disculpas propiamente tal sino constituye un elemento precursor que advierte la petición que vendrá.

Finalmente podemos señalar que los sujetos informantes de este estudio realizan el acto de nuclear de la petición con estrategias generalmente indirectas del tipo convencionalizadas, recurren a enunciados que contienen preguntas preparatorias 0 expresiones de posibilidad. Le preguntan al interlocutor sobre la posibilidad de cambiar la fecha de su examen, a través del verbo modal "poder" en presente y las formas impersonales "es posible". El acto principal es apoyado por dos modificadores principales: la justificación de la petición, que alude en casi todas las muestras a darle al profesor información sobre un problema personal, solicitando su empatía y comprensión. A este movimiento de apoyo, se le suma en ocurrencia la explicitación del problema que, en concreto, es el elemento que estimula la petición del estudiante, potenciando al acto nuclear e impidiendo que el interlocutor no acceda a la petición emitida.

\section{5- Reflexiones finales y proyecciones}

Si bien se han realizado publicaciones y diseñado materiales didácticos sobre la cercanía español-portugués respecto de la competencia lingüística que ha 


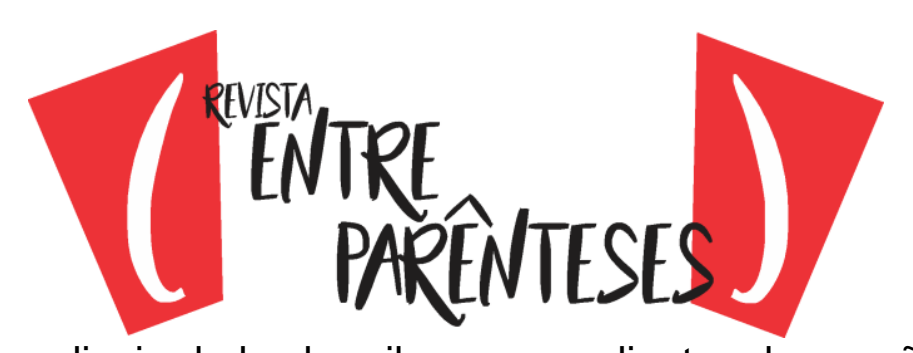

sistematizado el aprendizaje de los brasileros aprendientes de español, es imperativo el desarrollo de la habilidad pragmática, entendiendo que cada lengua y cultura tienen sus propios patrones de desenvolvimiento pragmático frente a la realización de actos de comunicación, en especial aquellos de alta ocurrencia, como es el caso de la petición.

Las producciones de los sujetos estudiados en este trabajo, arrojan una tendencia importante al uso de estrategias indirectas convencionalizadas para realizar una petición. A pesar de la cercanía personal que en general se les atribuye a los estudiantes brasileros con sus profesores, no quieren estratégicamente verse impositivos para ello recurren en la relación asimétrica (profesor-alumno) a formas modales principalmente con el verbo poder para realizar el acto nuclear o principal. Esto responde a que las peticiones amenazan la imagen del interlocutor como la del hablante y el estudiante no quiere invadir el territorio del interlocutor y menos si el profesor es quien ostenta el poder en la relación académica institucionalizada. A esto se suma que está en sus manos responder positivamente a la petición del estudiante.

Sin embargo, el hablante debe lograr su meta comunicativa de ahí que los movimientos de apoyo al acto principal, aparecen en gran parte de las realizaciones revisadas, formulados con recursos léxicos intensificadores. Los justificadores apuntan a que el interlocutor considere las razones de fondo de quien formula el acto de habla para acceder a su petición.

Se concluye que esto responde a la necesidad del hablante (el alumno) de equilibrar las estrategias y movimientos desplegadas, entre la atenuación y la intensificación, las llamadas "maniobras lingüísticas" (Escandell, 1995, p.33). Como reseñan Albelda y Barros (2018) ambas son estrategias complementarias que "pueden emplearse en un mismo hecho comunicativo para lograr una meta común en la interacción" (p.44)

Creemos que en el caso de estudiantes brasileros se sugiere además de la instrucción formal de algunos actos de comunicación de mayor complejidad y en diversos contextos, el contacto con hablantes nativos de diversas variedades del español, o bien, 


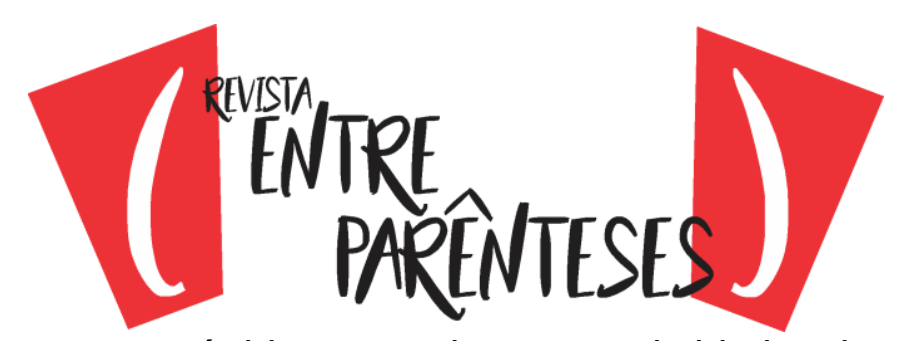

la estadía académica en un país hispanoparlante con el objetivo de concientizarlos sobre las sutilezas que diferencian las producciones de hablantes nativos y no nativos.

Dentro de las proyecciones respecto de este estudio exploratorio hemos considerado, en primer lugar, la ampliación del número de informantes para obtener un análisis más profundo. Con posterioridad, proponemos realizar un estudio contrastivo entre las realizaciones no nativas (portugués de Brasil) y hablantes nativos del español de Chile, desde un acercamiento pragmático intercultural e interlingüístico al acto de la petición.

\section{Referencias}

ALBELDA MARCO, Marta. Discordancia entre atenuación/cortesía e intensificación/descortesía en conversaciones coloquiales. En: BLAS ARROYO, José Luis; VELANDO CASANOVA, Mónica; CASANOVA AVALOS, Manuela (Eds.). Discurso y Sociedad. Contribuciones al estudio de la lengua en contexto social. Castellón de la Plana: Universidad de Castellón, 2005. p. 581-590

ALBELDA MARCO, Marta.; BARROS GARCÍA, María Jesús. La cortesía en la comunicación. Madrid: Arco/Libros, 2018.

BACHMAN, Lyle. Fundamental considerations in language testing. Oxford: Oxford University Press, 1990.

BACHMAN, Lyle F.; PALMER, Adrian S. Language Testing in Practice. Oxford: Oxford University Press, 1990.

BLUM-KULKA, Shoshana; HOUSE, Juliene.; KASPER, Gabriele. Cross-cultural pragmatics: requests and apologies. New Jersey: Ablex, 1989.

BLUM-KULKA, Shoshana; OLSHTAIN, Elite. Too many words: Length of utterance and pragmatic failure. Studies in Second Language Acquisition, 1986, 8 (2), p.165-179.

BRAVO, Diana. Categorías, tipologías y aplicaciones. Hacia una redefinición de la cortesía comunicativa. En: Bravo, Diana. (Ed.) Estudios de la (des) cortesía en español: Categorías conceptuales y aplicaciones a corpora orales y escritos. Estocolmo: Dunken, 2005, p. 21-52. 


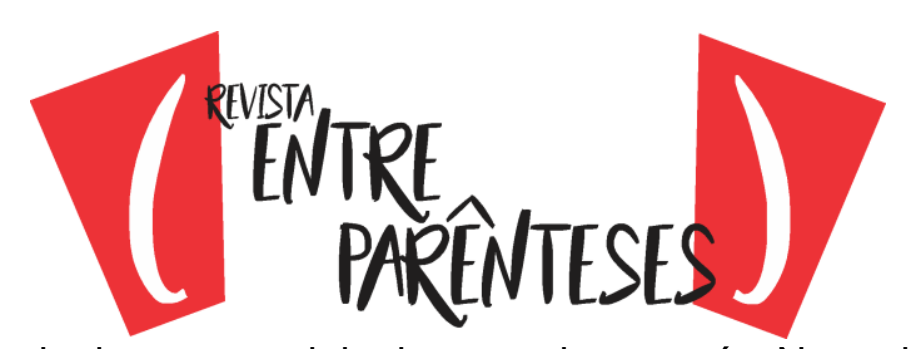

BRIZ, Antonio. Eficacia, imagen social e imagen de cortesía: Naturaleza de la estrategia atenuadora en la conversación cotidiana española. En: Bravo, Diana. (Ed.). Estudios de la (des) cortesía en español: Categorías conceptuales y aplicaciones a corpora orales y escritos. Estocolmo: Dunken, 2005, p. 53-91

BRIZ, Antonio; ALBELDA MARCO, Marta. Una propuesta teórica y metodológica para el análisis de la atenuación lingüística en español y portugués. La base de un proyecto en común (ES. POR. ATENUACIÓN). Onomázein, vol. 28, 2013, p.288-319.

CATHCART, Mercedes Causse; DE ALMEIDA, Lílian Rodrigues "¡No!" La negación en el español de Cuba y en el portugués brasileño bajo la perspectiva pragmática de la cortesía. Borealis - An International Journal of Hispanic Linguistics, vol. 3 (1), 2014, p. 83-102.

DA SILVA, Luiz Antônio. Cortesía y discurso académico. En: Briz, Antonio et al. (eds.). Actas III Coloquio Internacional Programa Edice, Departamento de Filología Española, Universidad de Valencia, 2008, p. 670-684.

DUARTE, Cristina Aparecida, CAMPOS, Simone Nascimento. Pídeme lo que quieras,

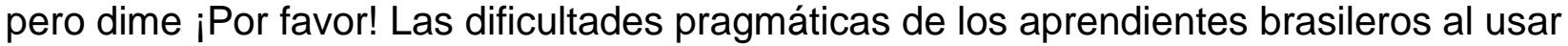
el Imperativo en español. Actas ASELE XV, 1999, p. 73-78.

ELIZAINCÍN, Adolfo. Contacto entre lenguas genéticamente emparentadas. El caso del español y del portugués. Signo y seña, v.6, 1996, p. 415-434.

ESCANDELL VIDAL, María Victoria. Cortesía, formulas convencionales y estrategias indirectas. Revista Española de Lingüística, vol. 25, (1), 1995, pp. 31-66. Disponible en

$<$ http://www.textosenlinea.com.ar/academicos/Cortesia\%20formulas\%20convencionale s\%20y\%20estrategias\%20indirectas.pdf> Acceso en junio 2020.

GALLARDO MORAES, Isabella. As manifestações de cortesia encontradas em emails empresariais. Português do Brasil e Espanhol peninsular: semelhanças e contrastes (tesis de maestría). Universidade de São Paulo, 2013. Disponible en: <<https://teses.usp.br/teses/disponiveis/8/8145/tde-01102014-180529/pt-br.php >>

Acceso en julio 2020.

GOFFMAN, Erving. Interactional ritual. Essays on face-to-face behavior. Nueva York: Doubleday, 1967.

KASPER, Gabriele. The role of pragmatics in language teacher education. En: BARDOVI HARLIG, Kathleen (Ed.). Beyond methods: Components of second language teacher education. Nueva York: McGraw-Hill, 1997, p. 113-136. 


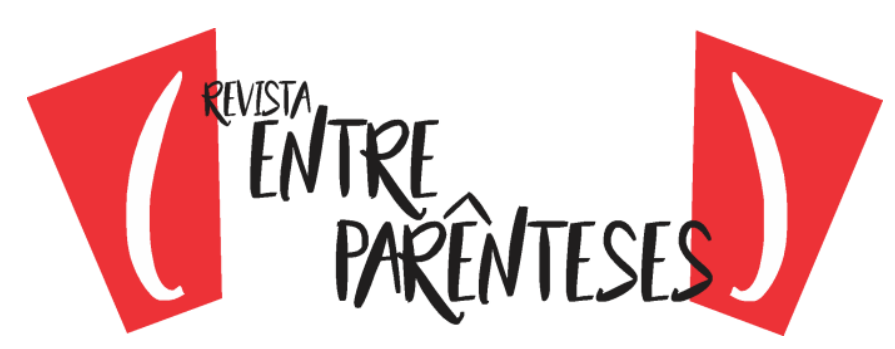

LADO, Robert. Linguistics across Cultures: Applied Linguistics for Language Teachers. Ann Arbor, Michigan: University of Michigan. 1957

LOPES RODRIGUES, Elisa Cristina; MARCELLE DE ANDRADE, Adriana. La cortesía verbal en los encuentros orales con fines de estudio: las estrategias lingüísticodiscursivas de atenuación en portugués y español. En: ESCAMILLA MORALES, Julio; HENRY VEGA, Grandfield (Eds.). Miradas Multidisciplinares a los fenómenos de cortesía y descortesía en el mundo hispánico. Barranquilla-Estocolmo: Universidad del Atlántico-Universidad de Estocolmo; CADIS - Programa EDICE, 2012. p. 369-391.

MORIONDO KULIKOWSKI, María Zulma; MAIA GONZÁLEZ, Neide. Español para brasileños. Sobre por dónde determinar la justa medida de una cercanía. Anuario brasileño de estudios hispánicos, v. 9, 1999, p.11-20.

OTERO BRAVO, María de Lourdes. Competencia pragmática e interferencias culturales en la enseñanza de español para alumnos brasileros. Actas XVI ASELE, 2005. p.501506.

RIENDA POLO, José; NIETO NÚÑEZ, Nuria María. Lingüística contrastiva y lenguas cognadas en el contexto del aula de ELE en Brasil. Porta Linguarum, v. 30, 2018. p.103115.

SEARLE, John. Speech acts. An essay in the philosophy of language. Cambridge: Cambridge University Press, 1969.

SERRANI-INFANTE, Silvana. Análise de ressonâncias discursivas em micro-cenas para estudo da identidade lingüístico-cultural. En: Trabalhos de Lingüística Aplicada, 24. Campinas: Universidade Estadual de Campinas, 1994. p.79-90.

THOMAS, Jenny. Cross cultural pragmatic failure. Applied Linguistics, vol. 4 (2),1983. p.91-112.

TOLEDO AZÓCAR, Sonia. Los Actos comunicativos en español como lengua extranjera. El caso del acto queja. Valparaíso, Chile: Ediciones Universitarias de Valparaíso, 2018.

VIGÓN ARTOS, Secundino. La enseñanza de la gramática del español como lengua extranjera a lusófonos. Actas XV ASELE, 2005. p. 903-913. 